\title{
3D modelling and visualization of large building using photogrammetric approach
}

\author{
Raad Awad Kattan*, Farsat Heeto Abdulrahman, Sami Mamlook Gilyana and Yousif Youkhna \\ Zaya \\ College of Engineering, University of Duhok, Iraq \\ Corresponding author: raadkattan@uod.ac
}

Submitted :06/11/2020

Revised :07/06/2021

Accepted : 27/06/2021

\begin{abstract}
The progress in modern technologies such as precise lightweight cameras mounted on unmanned aerial vehicles (UAV) and the more user-friendly software in the photogrammetric field allows for 3D model construction of any structure or shape. Software now achieves in sequence the processes of matching, generating tie points and block bundle adjustment and generating digital elevation models.

The aim of this study is to make a virtual 3D model of the College of Engineering, University of Duhok, Kurdistan Region, Iraq. The data input is vertical and oblique imagery acquired by UAV, with the ground control points being distributed on the surrounded ground, facades, and roof. Ground control points were measured by the GPS RTK system in addition to the reflectorless total station instrument. The data is processed mainly using Agisoft PhotoScan software as well as the Global Mapper and the ReCap software. The output is a 3D model, digital elevation model, and orthomosaic. Geometric and visual inspections were carried out. Some imperfections appeared on the sharp edges and parapets of the building. In the geometric accuracy of selected points on the building, the maximum standard deviation in the coordinates was $\pm 4 \mathrm{~cm}$. The relative accuracy in distance measurements was in the range from $0.72 \%$ to $4.92 \%$.
\end{abstract}

Keywords: 3D; Image; Modelling; Orthomosaic; Photogrammetry; UAV.

\section{INTRODUCTION}

It is common practice to use vertical aerial photographs or images taken by Unmanned Aerial Vehicles (UAV) or aircraft in topographic surveying to produce orthophoto mosaics, digital terrain models, and line maps. A complete imagery coverage of large buildings using the UAV is a new practice implemented in this study. The ability to tilt the UAV camera axis up or down with respect to the horizon produces oblique images that cover all the facades of any building, structure, or historical monuments.

The objective of this study is to establish a full 3D model of a 2000 sq.m building that is occupied by three departments of the College of Engineering, University of Duhok. The task was achieved through three stages. In the first stage, the coordinates of ground control points (GCPS) surrounding the building and on the building facades were measured and established. During the second stage, the aim was to obtain the required overlapped vertical and 
oblique images that cover all the building and its surrounding area. The third stage was to process the images using the Agisoft PhotoScan software, producing the 3D building model, and checking the geometrical accuracy.

Having assessed the produced model shape, geometrical accuracy level, and the work requirements, we gained experience by modelling any regular or irregular shape no matter how large or small it is.

\section{RELATED WORK}

(quan Li et al., 2016) used the Agisoft PhotoScan software to process the pictures captured using a nonmetric digital camera from a UAV with multiple angles. A multiview, three-dimensional reconstruction technique was adopted so as to establish a 3D model of the dream park rock landscape in Jiangxi province of China. The authors compared 10 distances measured on the model with their corresponding distances measured or calculated on the object. The relative errors ranged between $0.22 \%$ and $4.62 \%$.

(Akinade, 2020) investigated the accuracy attainable by using amateur cameras for point determination. For this purpose, a building façade was surveyed and processed by photogrammetric and conventional survey methods. The photogrammetric survey was completed using four different amateur cameras, and the conventional survey was carried out using a total station. A total of 37 control points and checkpoints were measured. The Agisoft PhotoScan photogrammetric software was used for processing and model generation. The root mean square error of the processed model coordinates was in the range of $\pm 5 \mathrm{~cm}$ for two of the used cameras.

(Jaud et al., 2016) investigated the level of accuracy that can be achieved using two software tools: Agisoft PhotoScan ${ }^{\circledR}$ Pro and an open-source alternative, IGNC MicMac ${ }^{\circledR}$, in rugged terrain, with poor GPS reception. A set of UAV images were taken, and several image processing tests were performed with different scenarios in order to analyze the sensitivity of each software package to different parameters. When computing the horizontal and vertical errors, both methods provided rather similar results. A precision of up to 3-4 $\mathrm{cm}$ was achievable with these software packages.

(Shashi et al., 2007) used Kodak CX 7300 digital camera and photogrammetric modeling software, PhotoModeler, in their works. They chose the corners of a building as a reference. A total of 35 camera positions were selected. They found that the RMS residual value in pixels achieved was less than 1.5 for all the points.

(Murtiyoso et al., 2017) used the DJI Phantom 3 Professional in their research. It is equipped with a $4 \mathrm{~mm}$ lens and a 6.5 x $5 \mathrm{~mm}$ sensor. They performed their test on the Josephine Pavilion building in the city of Strasbourg. An average distance of $8 \mathrm{~m}$ was used to take photos to cover the four facades. They took 555 images. 13 control and checkpoints were utilized. An average of $9 \mathrm{~mm}$ accuracy of the aerotriangulation was established, whereas the checkpoint precision was $7 \mathrm{~mm}$.

(Barrile et al., 2017) used Dji Phantom 2 Vision Plus drone to capture 371 photographic shots with an angle of field view $110^{\circ}$ or $85^{\circ}$. 10 control points were established using the Leica Viva GNSS GS15 rover in RTK mode. The study case was the surroundings of the Department of Agriculture Sciences. They used the Agisoft PhotoScan software to process the model. They reached average errors of $9 \mathrm{~cm}$ with a standard deviation $\sigma$ of $12 \mathrm{~cm}$.

A study was conducted by (Sanz-Ablanedo et al., 2018). A UAV was used to capture 2500 photos using 100 ground control points (GCPs) over an area of 1225 hectares. The Agisoft PhotoScan Professional software was used to process data. After analyzing the data, they found that using as many as possible GCPs can lead to more accurate results, especially in vertical control. They found that the Ground Sample Distance (GSD) was $6.8 \mathrm{~cm}$ on average, and the accuracy of the vertical component was approximately $10 \mathrm{~cm}$. 
Images from two flights, one with a camera mounted at nadir and the second with a $20^{\circ}$ angle, showed the benefits of increasing the accuracy of vertical control (Manfreda et al., 2019). DJI Phantom 4 Pro was used to collect data at an altitude of $60 \mathrm{~m}$. In their research, $16 \mathrm{GCPs}$ were distributed over an area of 2.7 hectares. Agisoft PhotoScan was used to process data, and the combination of two flights showed a vertical accuracy of $3.5 \mathrm{~cm}$ if more than six GCPs are used.

In the work of (Cryderman et al., 2014), UAV was used to capture 266 photos over a flying height of $118 \mathrm{~m}$. Data were processed and analyzed using Agisoft PhotoScan. A ground sample distance (GSD) of $2.5 \mathrm{~cm}$ was achieved and RSME of $44 \mathrm{~mm}$.

(Barry and Coakley, 2013) examined the accuracy of UAV image models by comparing them with a network of RTK-GPS. 45 ground markers were surveyed using RTK-GPS. Data were processed and analyzed using the Agisoft PhotoScan software. An accuracy of $6.8 \mathrm{~cm}$ was achieved. This accuracy is quite enough for preparing topographic maps, cadastral maps, and other surveying works.

\section{SITE DESCRIPTION}

The study is concerned with a 3D model of a 3-story building using the photogrammetric approach. The building occupies $2000 \mathrm{~m}^{2}$. Its height in one point is $13.75 \mathrm{~m}$. The total length of the building is $85 \mathrm{~m}$ with a width range between $19.70 \mathrm{~m}$ on one side and $43.3 \mathrm{~m}$ on another side. The building has a circular-shaped entrance and lobby with a diameter $=23.40 \mathrm{~m}$. On the upper roof of this part, there is a light transparent dome that is $7.5 \mathrm{~m}$ in diameter and $2.6 \mathrm{~m}$ in height. Air-condition system ducts and units in addition to water storage tanks occupy most of the building's upper roof surface. The building has 330 windows distributed on three of its facades.

Parts of the northern and eastern sides of the building were covered with tall trees that obscure imaging and control observations.

\section{CONTROL POINTS}

Nine ground control points surrounding the building were established. The points were clearly marked by $60 * 60$ $\mathrm{cm}$ aluminum targets. GPS observations were carried out to determine the coordinates of these points using Leica Viva GNSS, GS10 base receiver, and GS15 rover receiver. The system has a specified accuracy at a single base: \pm $8 \mathrm{~mm} \pm 1 \mathrm{ppm}$ for horizontal and $\pm 15 \mathrm{~mm} \pm 1 \mathrm{ppm}$ for the vertical component. In the study area, the GS10 GNSS receiver was set up on a known control (C12) point. The GS15 GNSS receiver was used as a rover for measuring and recording data on the 9 GCPs. The coordinate system used for measurements was the WGS84, UTM-38N projection.

A total of 92 self-adhesive $10 * 10 \mathrm{~cm}$ paper card targets (Figure 1a) were fixed as control points on the building's external walls and windows. The targets were distributed evenly to ensure good coverage of control points all over the building. The coordinates of these points were measured using the Leica TCR1101 total station in a reflectorless mode. According to the manufacture specifications, the instrument has an accuracy of $\pm 1.5^{\prime \prime}$ in angle measurements, distance accuracy of $\pm 3 \mathrm{~mm} \pm 2 \mathrm{ppm}$, and a measuring range $=80 \mathrm{~m}$ in the reflectorless mode.

The total station was positioned on the 9 GCPs (Figure 1b) from which the paper targets were observed and recorded using the SD card memory attached to the instrument. 
A total of 27 points were selected as GCPs on the upper roof surface of the building. Their coordinates were measured using the rover GS15 receiver. Points that can be clearly identified on the photos such as the parapet corner were selected.
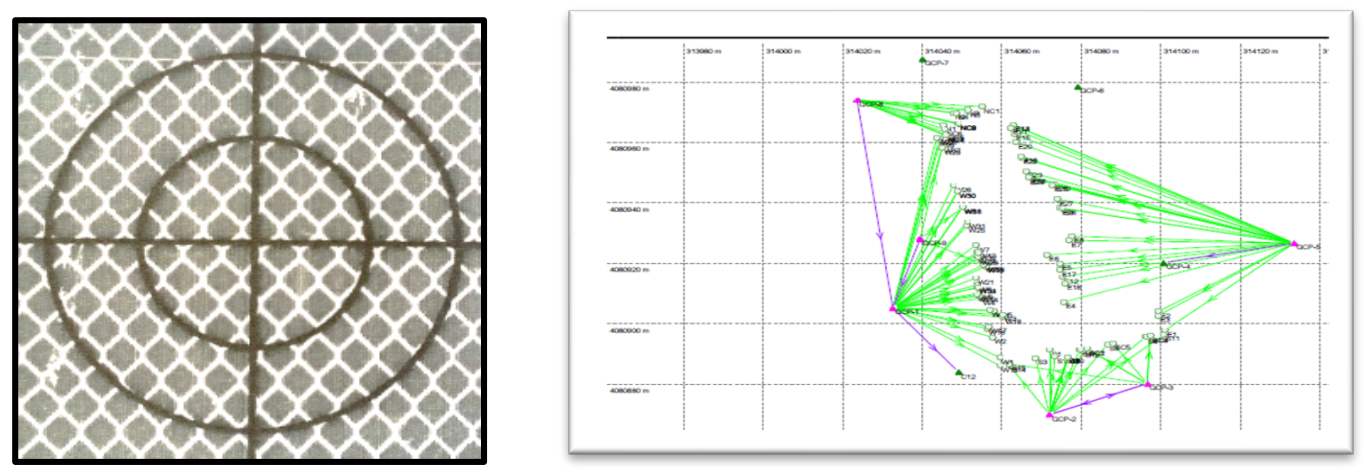

Figure 1a. Self-adhesive card target. Figure 1b. The distribution of the GCPs.

\section{FLIGHT PLANNING, IMAGING, AND PROCESSING}

The Phantom 4 Pro 4K-DJI was used as a platform for the imaging camera. The camera and the imaging parameters are shown in Table 1.

Table 1. Cameras and flight parameters.

\begin{tabular}{|c|c|}
\hline Camera Model & FC6310 (8.8mm) \\
\hline Resolution (pix) & $5472 \times 3648$ \\
\hline Focal Length & $8.8 \mathrm{~mm}$ \\
\hline Pixel Size & $2.41 \times 2.41 \mu \mathrm{m}$ \\
\hline Forward overlap & $70 \%$ \\
\hline Side overlap & $40 \%$ \\
\hline Ground resolution & $1.97 \mathrm{~cm} / \mathrm{pixel}$ \\
\hline
\end{tabular}

A total of 183 images were acquired for the building and the surrounded area. The software Pix4D Capture was used for flight planning. The coverage was acquired in 4 flights. Two flights were oblique, and the other two were vertical as shown in Figure 2. The first flight was set to take images at a distance of $35 \mathrm{~m}$ from the building facades at a height of $22 \mathrm{~m}$ from ground level with the camera depression angle of $22^{\circ}$ from the horizon. 


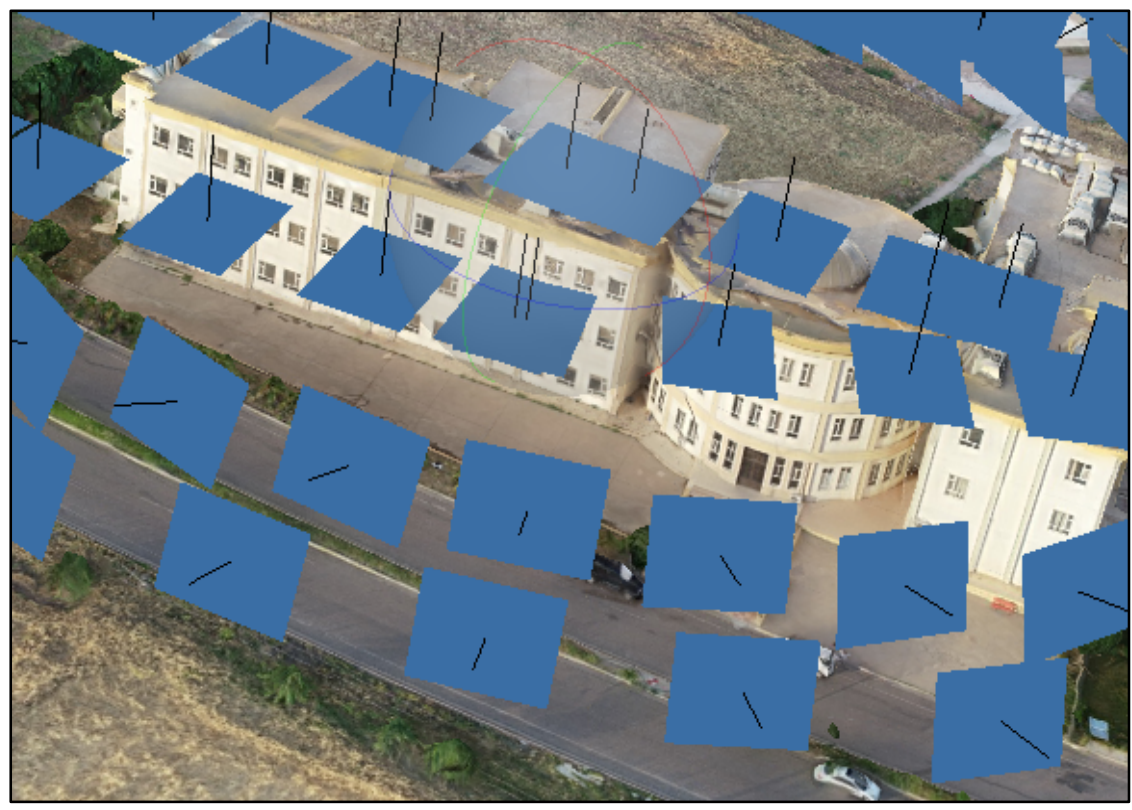

Figure 2. The 4 flight strips surrounding the building.

The Agisoft PhotoScan software was used to process the data and acquired the building 3D model. The input was the 183 images and the 128 GCPs (markers). The processing flow diagram is shown in Figure 3. One step in constructing a 3D model in Agisoft PhotoScan software and after aligning the photos is the selection of the control points on the overlapped images. On selecting the point on the first image, the epipolar line will appear passing through the corresponding point on the second image (Figure (3.9)). This will indicate the degree of matching accuracy.

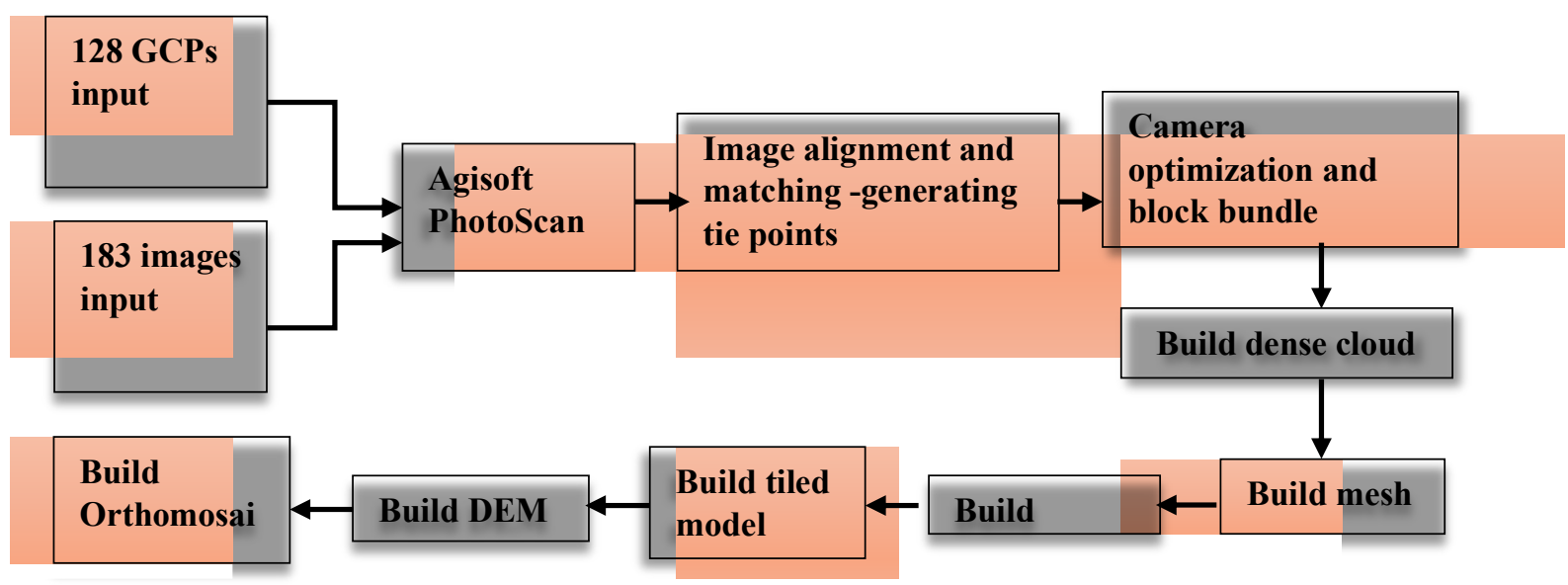

Figure 3. The Agisoft PhotoScan working flow diagram.

Table 2 shows the generated data details. 
Table 2. Details of the generated data.

\begin{tabular}{|l|l|}
\hline Tie points & 190,296 points \\
\hline Dense cloud & $21,979,642$ points with medium quality \\
\hline 3D model & 89,893 faces \\
\hline Tiled model & $1.19 \mathrm{~cm} /$ pix \\
\hline DEM & $7228 \times 7228 \quad 4.74 \mathrm{~cm} /$ pix \\
\hline Orthomosaic & $28795 \times 28835 \quad 1.19 \mathrm{~cm} / \mathrm{pix}$ \\
\hline
\end{tabular}

Figure 4 shows the Agisoft PhotoScan active window. The window can show the selected image, the model, workflow, images perspective centers, and GCPs details.

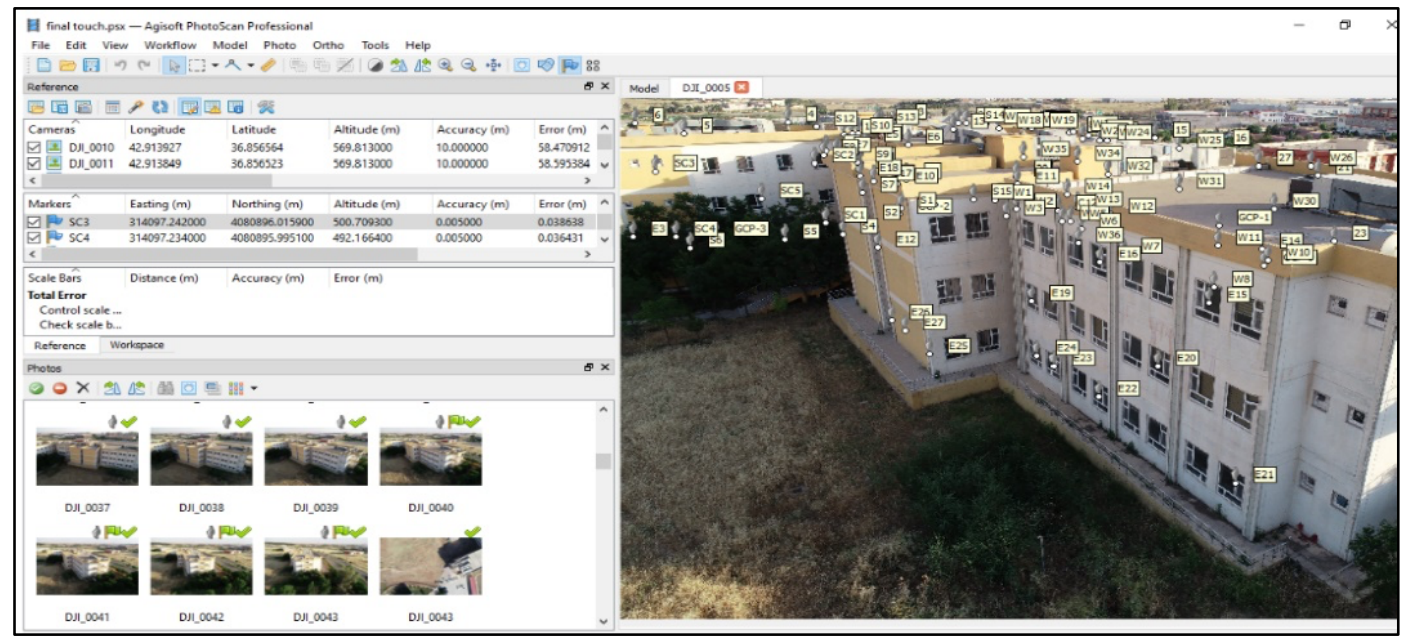

Figure 4. The Agisoft PhotoScan active window.

Table (3) shows the final root mean square error report in the 128 GCPs achieved after several stages of refinements by adding control points, applying block bundle adjustment, and camera calibration parameters. The total RMSE does not exceed $\pm 5 \mathrm{~cm}$, a value that is reasonable relative to the size and extent of the project.

Table 3. RMSE of the measured GCPs after refinement stages.

\begin{tabular}{|c|c|c|c|c|c|c|}
\hline Count & $\begin{array}{c}\text { X error } \\
(\mathbf{c m})\end{array}$ & $\begin{array}{c}\text { Y error } \\
(\mathbf{c m})\end{array}$ & $\begin{array}{c}\text { Z error } \\
(\mathbf{c m})\end{array}$ & $\begin{array}{c}\text { XY error } \\
(\mathbf{c m})\end{array}$ & $\begin{array}{c}\text { Total } \\
(\mathbf{c m})\end{array}$ & $\begin{array}{c}\text { Image } \\
(\mathbf{p i x})\end{array}$ \\
\hline $\mathbf{1 2 8}$ & \pm 3.44 & \pm 2.60 & \pm 1.89 & \pm 4.31 & \pm 4.71 & \pm 0.49 \\
\hline
\end{tabular}

The medium quality process of the dense cloud took approximately 2 hours. Figure 5a shows the tiled model, while Figure $5 b$ shows the tiled model with a GCPs marker. 

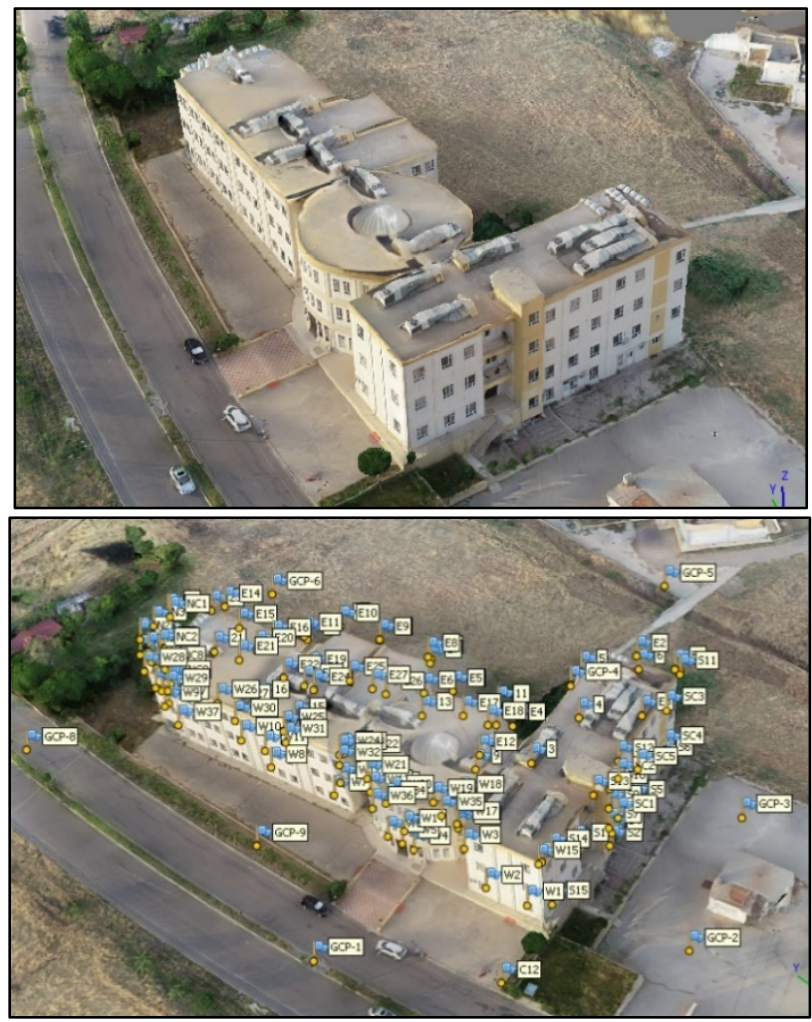

Figure 5a. The finished tiled model. Figure 5b. The model with control points.

Most of the building details appeared perfectly well. In Figure 6a, however, limited deformations have been noticed on some straight edges like top roof parapets and some corners (Figure 6b). Obstructions like trees (Figure 6c) prevent overlapping images and control measurements. Details behind these obstructions appear largely deformed.
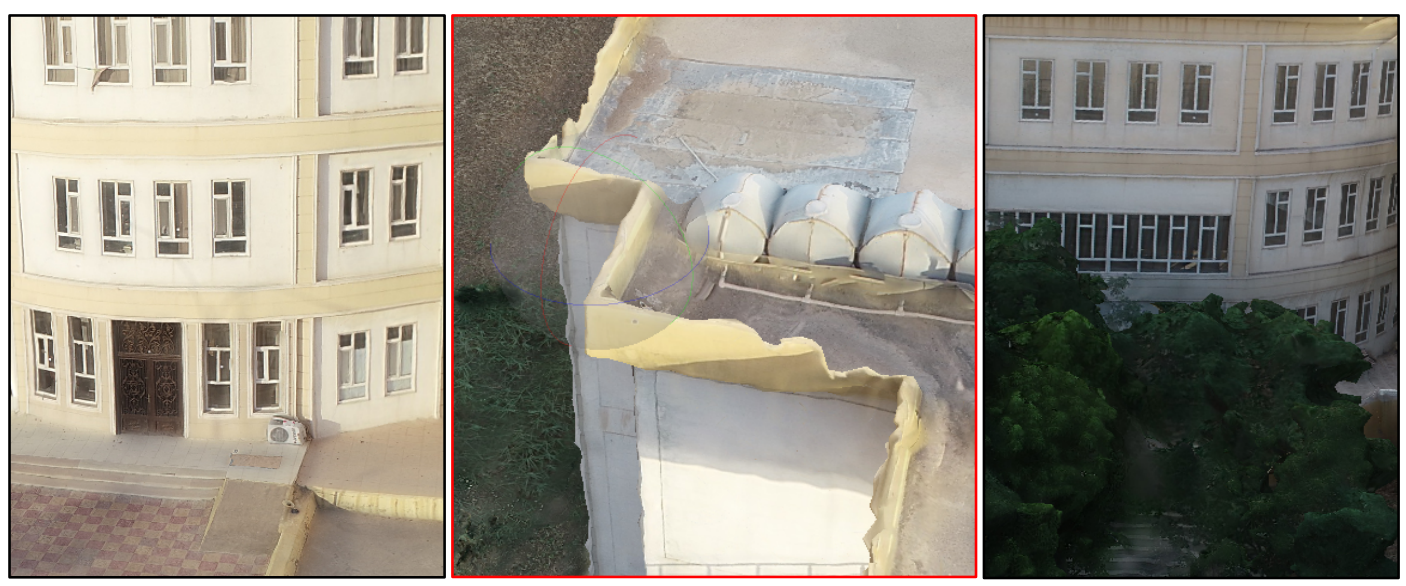

Figure 6a. Part of the model showing the apparent model quality. Figure 6b. Parapet and corners distortions.

Figure. 6c. Obstruction by trees. 
Figure 7a shows the part of the building top view with medium quality dense cloud. Figure $7 \mathrm{~b}$ shows the same view but processed in ultra-high-quality dense cloud. On changing to ultra-high-quality processing, there is a clear improvement in image fidelity. However, distortion in the building parapets was also obvious.

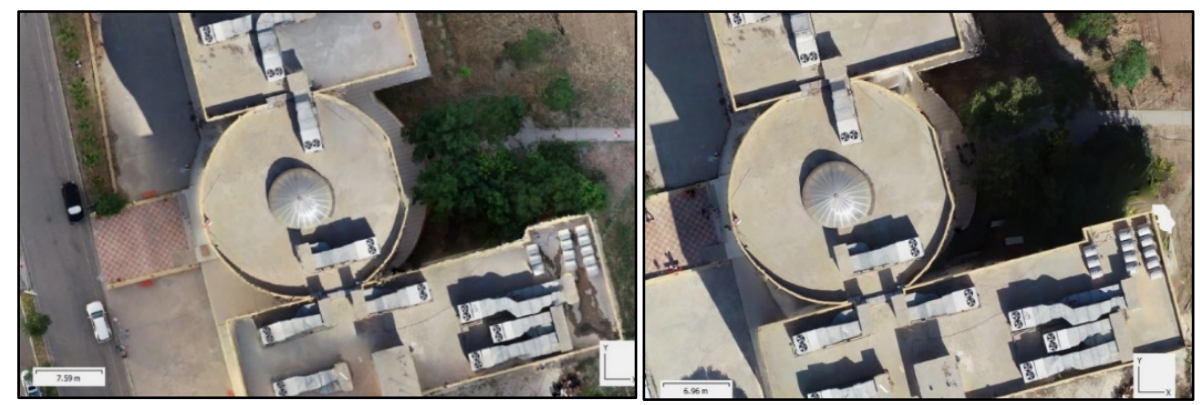

Figure 7a. Medium quality dense cloud model. Figure 7b. Ultra-high-quality model.

The PhotoScan allows building a DEM (Figure 8a) with a point density of $7228 \times 7228$ at a resolution of $4.74 \mathrm{~cm} /$ pix. Different colors are assigned to different elevation ranges. Contour lines are added to the DEM at $1 \mathrm{~m}$ intervals. Figure $8 \mathrm{~b}$ shows the orthomosaic generated with $28795 \times 28835$ pixels at a resolution of $1.19 \mathrm{~cm} /$ pix. Contour lines are also added to the mosaic at $1 \mathrm{~m}$ intervals.
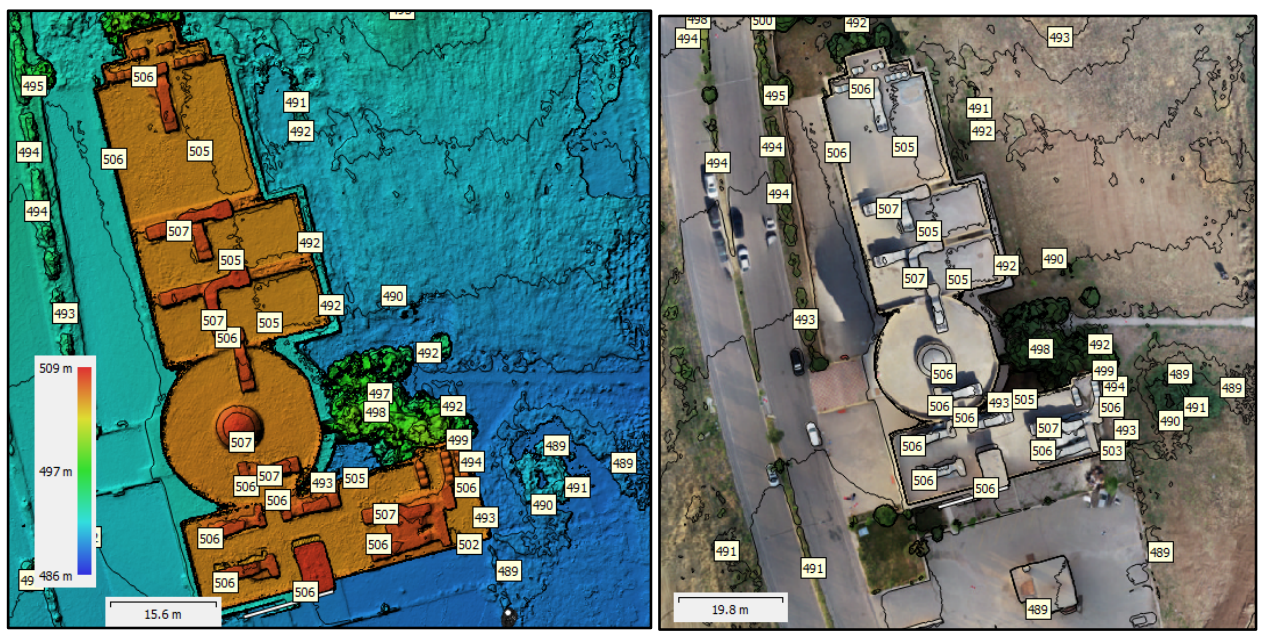

Figure 8a. PhotoScan color coded DEM with contour lines. Figure 8b. The orthomosaic.

A profile can be generated by drawing a polyline across any part of the DEM or the orthomosaic in the desired position, line $\mathrm{AB}$ Figure 9a, producing the profile in different colors for different elevation ranges. 

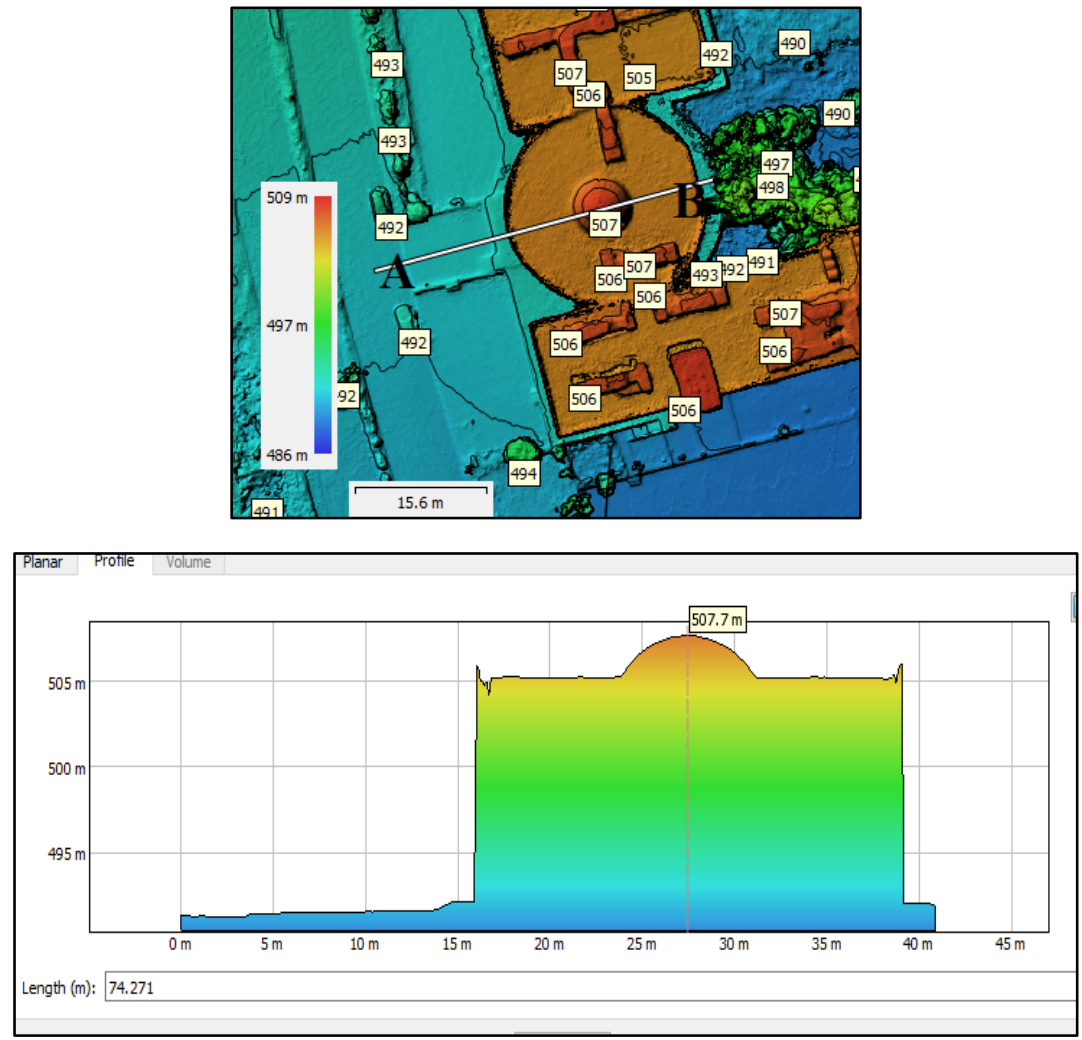

Figure 9a. Location of the profile AB. Figure 9b. The produced profile with color coded.

Vertical walls of the building produce parallel contour lines, which appear in the model perspective view. These contours are not diffused with the natural ground contours. Figure 10a shows the building western façade with $1 \mathrm{~m}$ interval contour lines. Further orbiting the model will show the contours of the other side of the building plus the natural ground contour lines behind the building (Figure 10b). The building transparency might be considered as a software drawback to be treated.

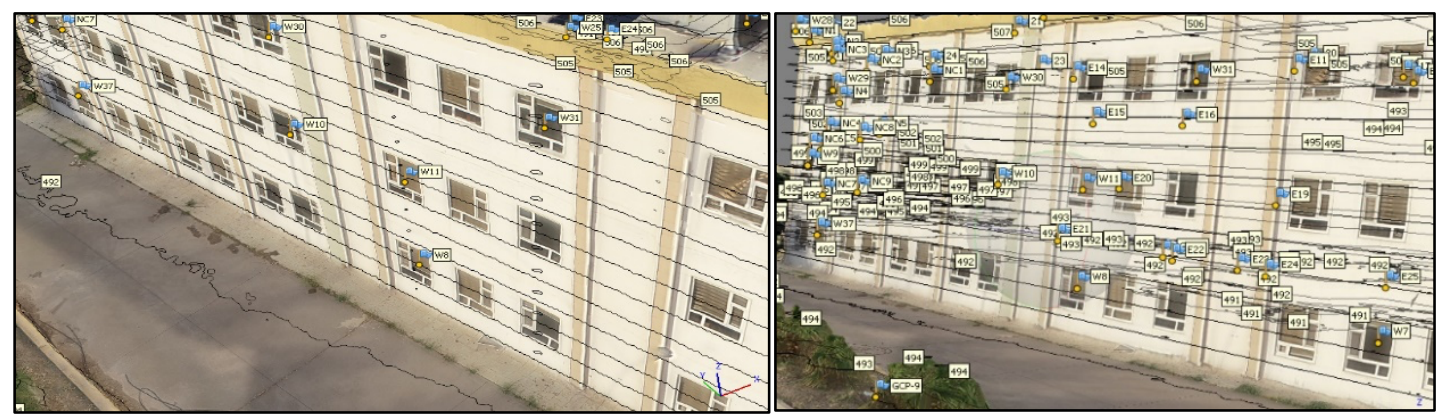

Figure 10a. Tiled model with $1 \mathrm{~m}$ contour lined.

Figure 10b. The back façade contour lines appear on the front façade.

The DEM was exported with the same point density of $7228 \times 7228$ and the same resolution of $4.74 \mathrm{~cm} / \mathrm{pix}$ in a TIFF format to the Global Mapper software producing a more enhanced texture image (Figure 11a). Figure 11b shows a $3 \mathrm{D}$ view produced by the same software using the DEM data only and height codes. 

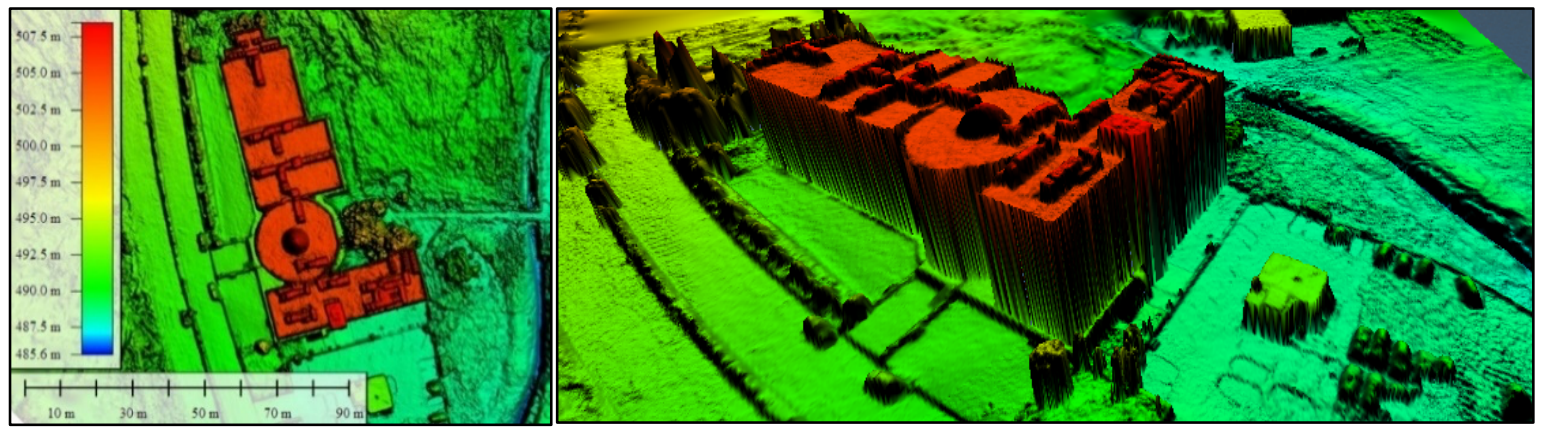

Figure 11a. Global Mapper produced color coded DEM. Figure 11b. 3D view.

The generated dense cloud is saved in ASPRS LAS (*. las) format and exported to the Autodesk ReCap program. In the ReCap software, a 3D model is produced in different forms. Figure 12a shows the model built with specified colors of points based on height. Figure $12 \mathrm{~b}$ shows the model built with specified colors of points based on the intensity value.
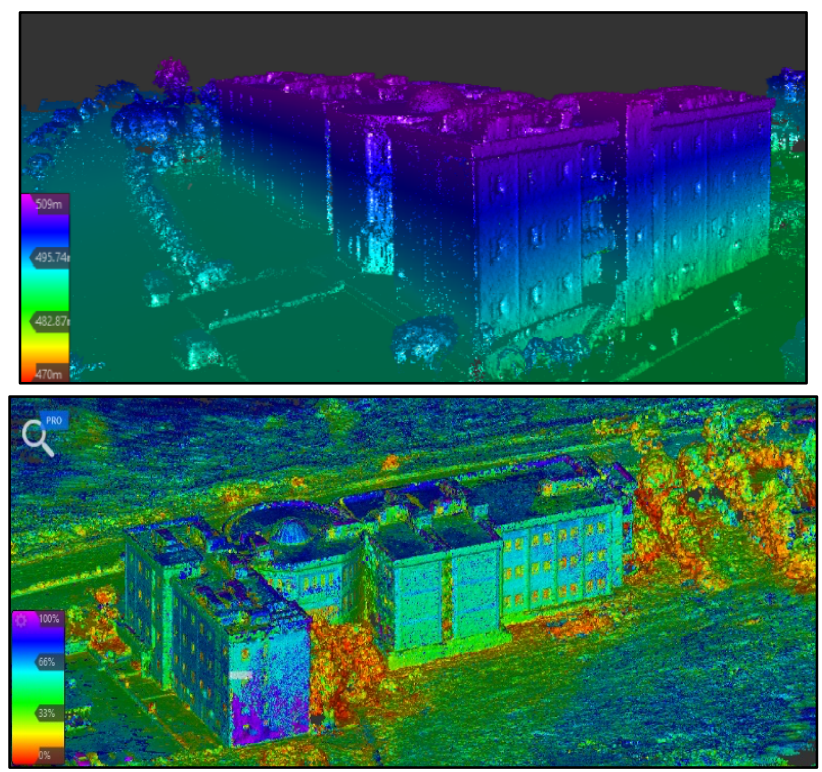

Figure 12a. ReCap produced 3D view with colored coded heights.

Figure 12b. The model with light intensity color coded.

\section{ACCURACY CHECK}

As a mean to check the geometrical accuracy, the 3D model generated by the ReCap software was used. It was possible to locate the cursor at any point on the model and read its coordinates, as shown in the margin of Figure 13. These coordinates were compared with the measured GPS coordinates. It was possible to identify 8 control points on different building facades. The discrepancy between the two sets of coordinates is tabulated in Table (4). The standard deviation in any of the 3 three coordinates does not exceed $\pm 4 \mathrm{~cm}$. 


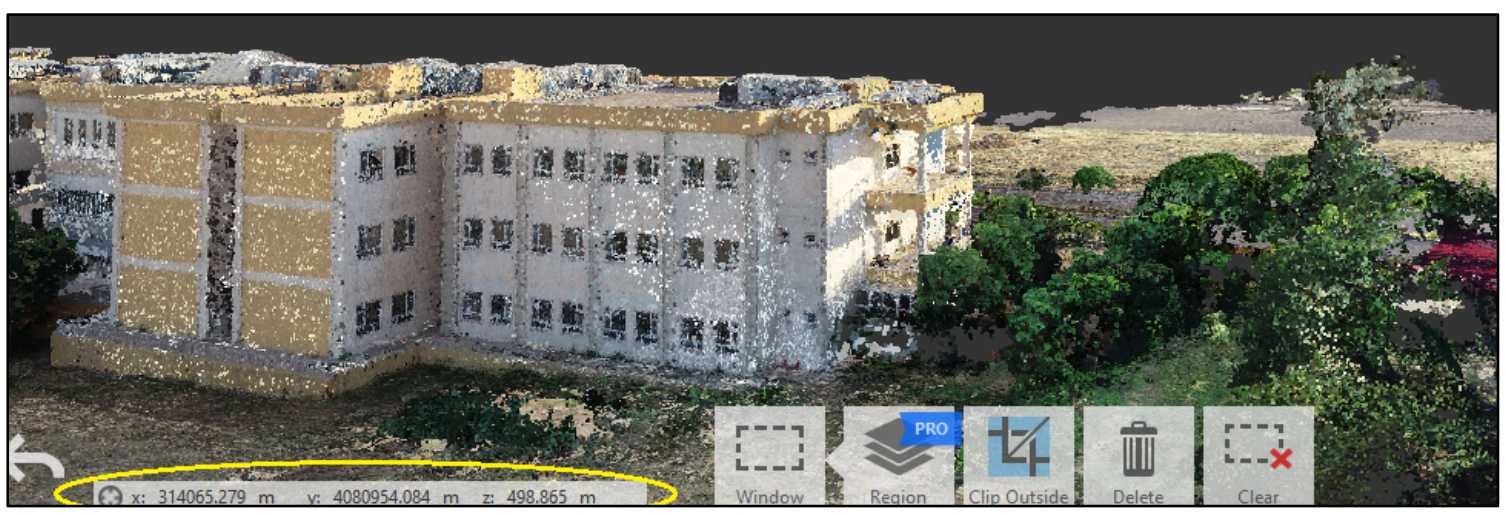

Figure 13. ReCap generated 3D model allowing to measure the coordinates of any point on the facades.

Table 4. Discrepancy between the GPS coordinates and ReCap model coordinates.

\begin{tabular}{|c|c|c|c|c|c|c|c|c|c|}
\hline Point & $\mathrm{E}(\mathrm{GPS})^{* *}$ & $\mathrm{~N}(\mathrm{GPS})$ & $\mathrm{h}(\mathrm{GPS})$ & $\mathrm{E}^{*}$ & $\mathrm{~N}^{*}$ & $\mathrm{~h}^{*}$ & $\Delta \mathrm{E}$ & $\Delta \mathrm{N}$ & $\Delta \mathrm{h}$ \\
\hline E17 & 314074.608 & 4080917.805 & 74.616 & 314074.616 & 4080917.859 & 501.78 & 0.008 & 0.054 & -0.004 \\
\hline E19 & 314066.646 & 4080948.467 & 66.655 & 314066.655 & 4080948.482 & 497.978 & 0.009 & 0.015 & 0.04 \\
\hline S1 & 314072.003 & 4080891.479 & 72.016 & 314072.016 & 4080891.465 & 493.867 & 0.013 & - & -0.013 \\
\hline SC3 & 314097.242 & 4080896.016 & 97.181 & 314097.181 & 4080896.027 & 500.755 & -0.061 & 0.011 & 0.046 \\
\hline SC4 & 314097.234 & 4080895.995 & 97.239 & 314097.239 & 4080895.985 & 492.142 & 0.005 & -0.01 & -0.024 \\
\hline W37 & 314045.044 & 4080958.751 & 45.037 & 314045.037 & 4080958.676 & 494.267 & -0.007 & - & 0.075 \\
\hline W7 & 314053.316 & 4080926.048 & 53.327 & 314053.327 & 4080926.071 & 494.252 & 0.011 & 0.023 & 0.021 \\
\hline W8 & 314050.11 & 4080938.586 & 50.15 & 314050.15 & 4080938.562 & 494.063 & 0.04 & - & -0.021 \\
\hline & & & & & & St. dev. & \pm 0.029 & \pm .038 & \pm 0.027 \\
\hline
\end{tabular}

*Coordinates measured on the 3D model generated by the Autodesk ReCap software depending on the imported dense cloud generated by Agisoft PhotoScan. ** All measurements are in meters.

Another geometrical accuracy check was carried out by measuring five distances directly on the building by tape, and these distances were compared with distances measured on the profiles created using the Global Mapper.

Figures $14 \mathrm{a}$ and $14 \mathrm{~b}$ show the location and distances of the profile $\mathrm{AB}$, which are measured on the Global Mapper profile. Figure 15a shows the location of the profile CD in an outside stair on the south side of the building. One height marked with the number 5 is measured on the model and directly on the building. Results of these measurements are given in Table 5. 

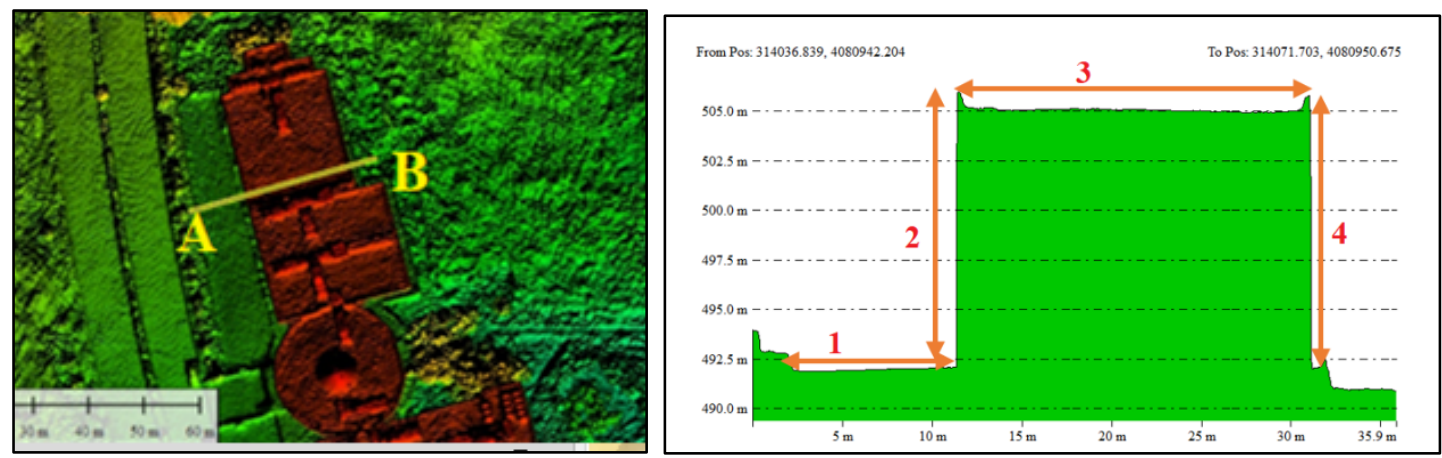

Figure 14a. Position of the profile line AB on Global Mapper model. Fig. 14b. The produced profile.
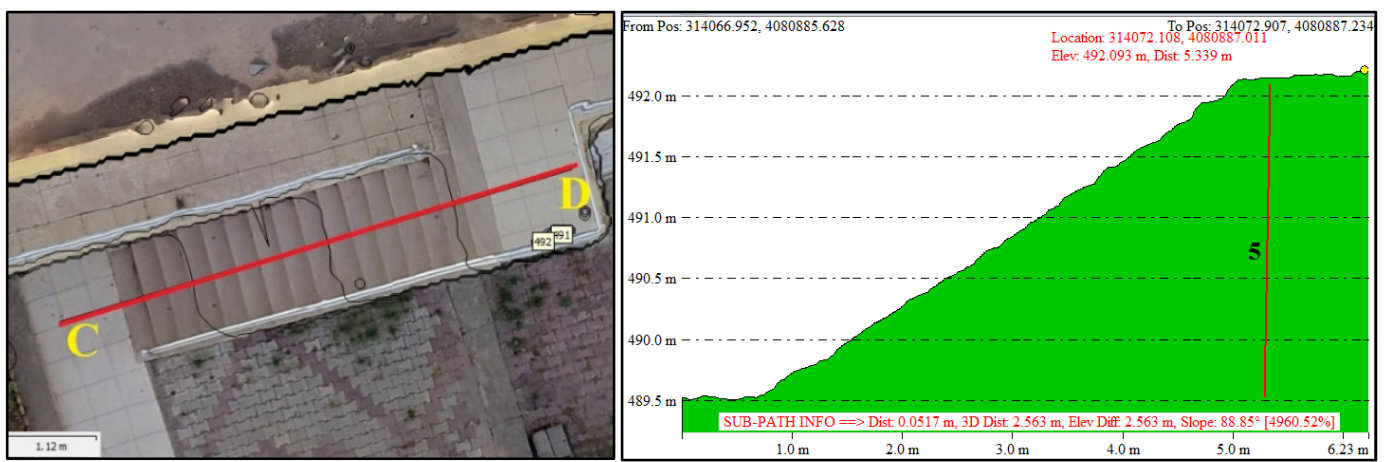

Figure 15a. The location of the profile line CD. Fig. 15b. The produced stair profile.

Table 5. Discrepancies of five distances measured directly and on the model.

\begin{tabular}{|c|c|c|c|c|}
\hline Distance & Direct (m) & Model distance (m) & Discrepancy (cm) & Relative discrepancy \\
\hline 1 & 9.98 & 9.91 & -7 & $-0.71 \%$ \\
\hline 2 & 13.95 & 13.85 & -10 & $-0.72 \%$ \\
\hline 3 & 19.81 & 19.73 & -8 & $-0.41 \%$ \\
\hline 4 & 13.95 & 13.86 & -9 & $-0.65 \%$ \\
\hline 5 & 2.51 & 2.64 & 13 & $4.92 \%$ \\
\hline
\end{tabular}

Table 5 shows that the discrepancies between the tape measured distances and the Global Mapper profile distance range between 7 and 13 with a relative error range from $0.41 \%$ to $4.91 \%$. 
Examining the stair profile (Figure 15b), it is clear that the steps that had a height of $18 \mathrm{~cm}$ did not appear clearly on the profile. Repeating the model processing but with ultra-quality in dense cloud generation, a profile, shown in Figure 16, is produced

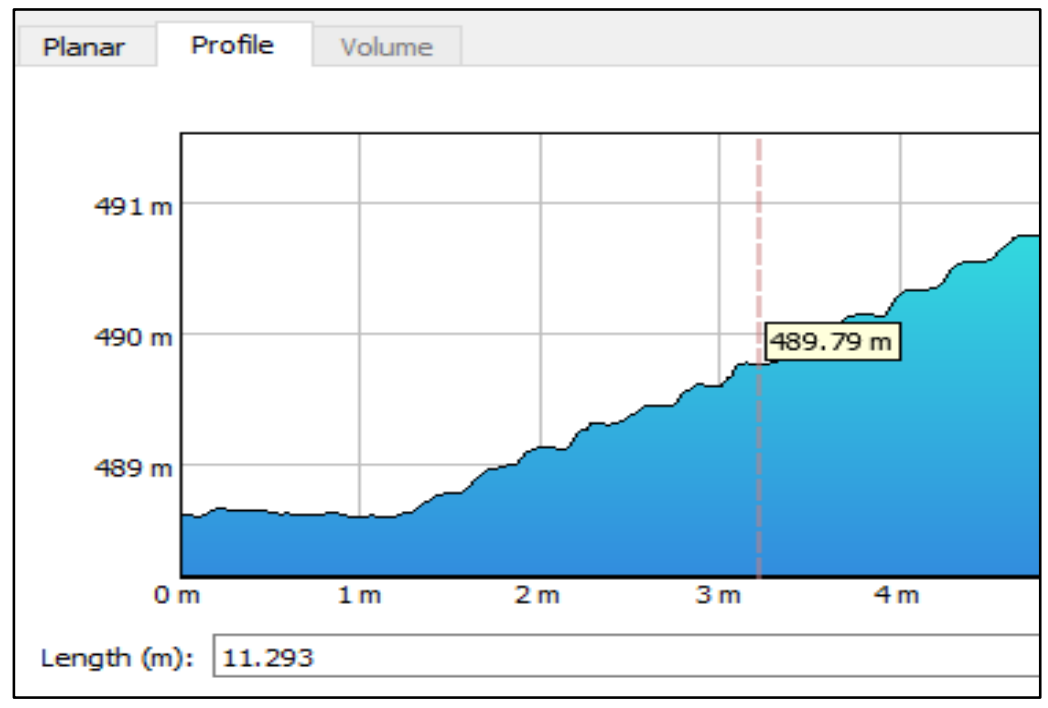

Figure 16. Stair profile produced using ultra-high-quality dense cloud.

Figure 16 shows the appearance of the stair steps; although it shows irregularity in shape to some extent, the average height of the steps was $17 \mathrm{~cm}$ measured on the model, which is not far away from the actual $18 \mathrm{~cm}$ height.

\section{DISCUSSION AND CONCLUSIONS}

1- Regular shape buildings require vertical (nadir) and oblique image coverage. Model distortion is expected on the lack of vertical coverage

2- Geometrical accuracy check was carried out by comparing the GPS coordinates of 8 points located on the building facades with their coordinates on the model generated by Agisoft PhotoScan and measured on the Autodesk recap software. The check reveals that the maximum standard deviation in $\mathrm{E}, \mathrm{N}$, and $\mathrm{h}$ was less than $\pm 4 \mathrm{~cm}$.

3- Geometrical accuracy check was also carried out by comparing 5 distances directly tapped on the building and on profiles that were generated by Global Mapper software depending on the Agisoft PhotoScan DEM. The check reveals that, for four distances, the relative discrepancy was less than $0.72 \%$. For one distance, it was $4.92 \%$.

4- On adding GCPs for the process of georeferencing in the PhotoScan, the images on which the points are being selected display all the front side and backside control points. Figure 17 shows the building's east façade image under processing and control point's location. In addition to the east side control points, the control points of the west and south facades appear as well. The research team noticed that the same remark took place on the building model. This point might be considered as a drawback in the software design to be treated, as the model appears transparent, showing all sides of the control points. 


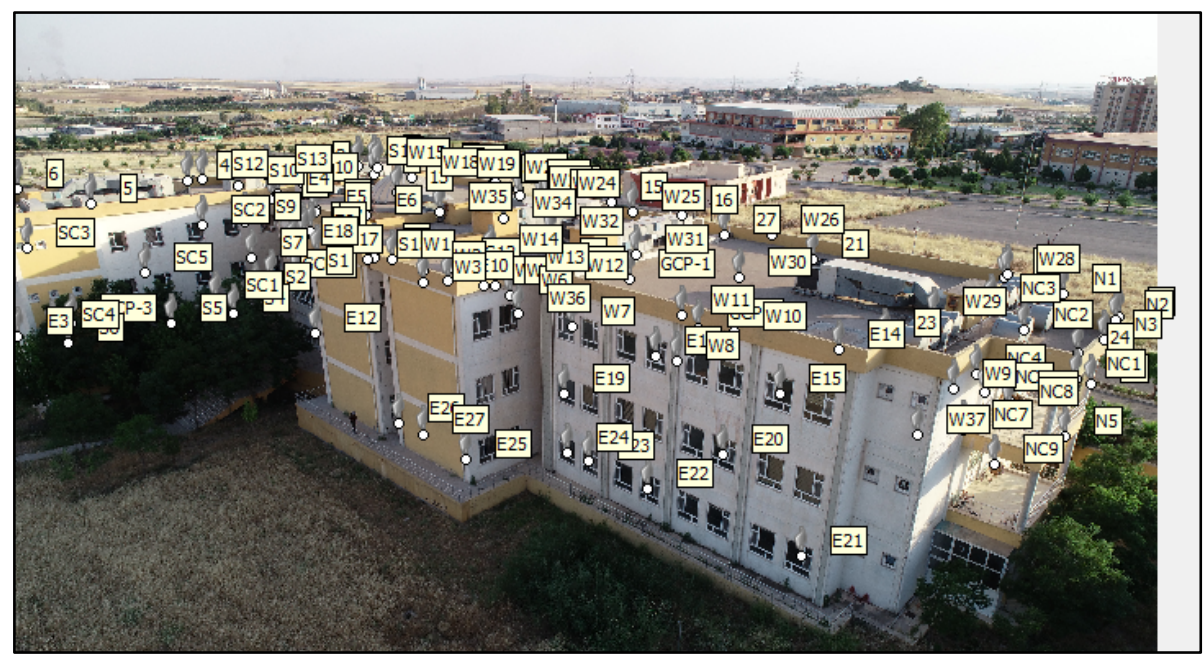

Figure 17. The east side control points; the control points of the west and south facades appear as well.

5- As an alternative for using the reflectorless total station instrument with the GCPs to measure on facades control points, the RTK receiver can be mounted on board to the UAV to obtain more accurate exposure center coordinates to be used in the intersection process. This will reduce the required number of GCPs.

6- As a step towards overcoming model deformation due to obscure details, handheld cameras can be used to capture photos of facades obstructed by trees.

\section{REFERENCES}

AKINADE, B. J. W. S. N. 2020. Investigation of the Accuracy of Photogrammetric Point Determination Using Amateur/Non-Netric Cameras. 145, 298-312.

BARRILE, V., BILOTTA, G., NUNNARI, A. J. I. A. O. T. P., REMOTE SENSING \& SCIENCES, S. I. 2017. 3D modeling with photogrammetry by UAVs and model quality verification. 4, 129.

BARRY, P. \& COAKLEY, R. 2013. Accuracy of UAV photogrammetry compared with Network RTK GPS, Baseline Surveys Ltd.

CRYDERMAN, C., MAH, S. B. \& SHUFLETOSKI, A. J. G. 2014. Evaluation of UAV photogrammetric accuracy for mapping and earthworks computations. 68, 309-317.

JAUD, M., PASSOT, S., LE BIVIC, R., DELACOURT, C., GRANDJEAN, P. \& LE DANTEC, N. J. R. S. 2016. Assessing the accuracy of high resolution digital surface models computed by PhotoScan ${ }^{\circledR}$ and MicMac ${ }^{\circledR}$ in sub-optimal survey conditions. 8, 465.

MANFREDA, S., DVORAK, P., MULLEROVA, J., HERBAN, S., VUONO, P., ARRANZ JUSTEL, J. J. \& PERKS, M. J. D. 2019. Assessing the accuracy of digital surface models derived from optical imagery acquired with unmanned aerial systems. 3,15 .

MURTIYOSO, A., KOEHL, M., GRUSSENMEYER, P., FREVILLE, T. J. I. A. O. P., REMOTE SENSING \& SCIENCES, S. I. 2017. Acquisition And Processing Protocols For Uav Images: 3d Modeling Of Historical Buildings Using Photogrammetry. 4. 
QUAN LI, X., AN CHEN, Z., TING ZHANG, L. \& JIA, D. J. P. E. S. 2016. Construction and accuracy test of a 3D model of non-metric camera images using Agisoft PhotoScan. 36, 184-190.

SANZ-ABLANEDO, E., CHANDLER, J. H., RODRÍGUEZ-PÉREZ, J. R. \& ORDÓÑEZ, C. J. R. S. 2018. Accuracy of unmanned aerial vehicle (UAV) and SfM photogrammetry survey as a function of the number and location of ground control points used. 10, 1606.

SHASHI, M., JAIN, K. J. A. J. O. E. \& SCIENCES, A. 2007. Use of photogrammetry in 3D modeling and visualization of buildings. 2, 37-40. 